\title{
Improving transfusion safety through Social Networking Sites
}

\author{
Nakhli M.S. ${ }^{1}$, Kahloul M. ${ }^{1}$, Kacem I. ${ }^{2}$, Boughatas W. ${ }^{2}$, Mhamdi S. ${ }^{1}$, Mrizak N. ${ }^{2}$ \\ ${ }^{1}$ Sahloul Hospital, Dept of Anaesthesiology \& Intensive Care, Sousse, Tunisia, \\ ${ }^{2}$ Farhat Hached Hospital, Department of Occupational Medicine, Sousse, Tunisia
}

\section{Introduction:}

Transfusion safety requires the adhesion of clinicians to the best transfusion practices. Several studies evaluating the level of transfusion safety knowledge and practices found disappointing results despite the efforts of faculty staff. Social networking sites (SNS) such as Facebook $\AA$, while not originally intended to be used as learning environments, may be adapted for distance-learning programs [1]. The aim of this study is to evaluate the impact of a training program through the Facebook social network, on young physicians' knowledge of blood transfusion safety.

\section{Methods:}

This is an interventional study carried out in Sahloul teaching hospital over a 3 months period. The first stage of the study was an initial assessment of knowledge through a questionnaire. Subsequently, a group was created on 'Facebook' including the study population (interns and residents of anesthesia and intensive care and surgical specialties).

Key messages were broadcast on this group in the form of a caricature over a period of 1 month. Finally, the survey was repeated after completion of the training program for 1 month.
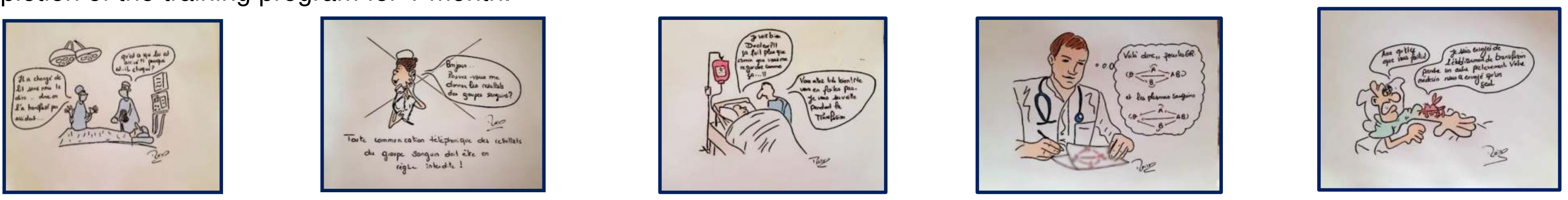

\section{Results:}

\section{Discussion:}

The overall response rate to the survey was $77 \%$. The mean age of the study population was $27,5 \pm 2,9$ years with a sex ratio of 0.7 .

Our population was divided into 39 internal and 38 residents. They were affected mainly in anesthesia-intensive care department in $44.2 \%$ of cases. The transfusion rate was more than twice a week in $63.6 \%$ of cases.

The correct response rate by question before the training program was $52.33 \pm 15 \%$. Sixty seven percent of our participants correctly answered more than half of the questions.

The correct response rate by question after the intervention was 66.12 $\pm 13.7 \%$ ( $p<10-3)$. Almost the majority $(92.2 \%)$ of participants had correctly answered more than half of the questions.

Our participants rated this evaluation program as beneficial in $70 \%$ of the cases (Table 1).

Table1 : Evolution of participants correct answers by question

\begin{tabular}{|lccc|}
\hline & \multicolumn{2}{c}{ Group } & P \\
\cline { 2 - 4 } & $\begin{array}{c}\text { Before } \\
\text { intervention }\end{array}$ & $\begin{array}{c}\text { After } \\
\text { intervention }\end{array}$ \\
\hline Question 1 & 33 & 33 & 0.77 \\
Question 2 & 56 & 70 & $<\mathbf{1 0 ^ { - 3 }}$ \\
Question 3 & 53 & 65 & $\mathbf{0 . 0 0 3}$ \\
Question 4 & 34 & 63 & $<\mathbf{1 0 ^ { - 3 }}$ \\
\hline Question 5 & 72 & 72 & 0.1 \\
Question 6 & 48 & 64 & $<\mathbf{1 0}$ \\
Question 7 & 23 & 19 & 0.6 \\
Question 8 & 12 & 12 & 0.88 \\
\hline Question 9 & 48 & 48 & 0.66 \\
Question 10 & 22 & 41 & $\mathbf{0 . 0 0 1}$ \\
\hline Question 11 & 12 & 35 & $<\mathbf{1 0}$ \\
Question 12 & 52 & 63 & $\mathbf{0 . 0 0 7}$ \\
Question 13 & 10 & 19 & $\mathbf{0 . 0 4 3}$ \\
Question 14 & 73 & 70 & 0.75 \\
\hline Question 15 & 39 & 46 & 0.12 \\
Question 16 & 49 & 44 & 0.67 \\
Question 17 & 40 & 43 & 0.39 \\
Question 18 & 52 & 62 & $\mathbf{0 . 0 1 3}$ \\
\hline Question 19 & 52 & 54 & 0.38 \\
Question 20 & 27 & 49 & $<\mathbf{1 0}-\mathbf{3}$ \\
\hline
\end{tabular}

References:

1. Pinto $A$ and al. E-learning tools for education: regulatory aspects, current applications in radiology and future prospects. Radiol Med. 2008.

2. D Scanfeld. Dissemination of health information through social networks: Twitter and antibiotics. Am J Infect Control. 2010.

3. Pickering JD and al. Medical student use of Facebook to support preparation for anatomy assessments. Anat Sci Educ. 2017

Social Media (SM) is an Internet application in which users generate the material. Facebook, Twitter or YouTube user's determine what is published via posts, status updates and videos. SM also helps users make connections by suggesting other users with whom one might want to be 'friends'.

Of adults that use Internet in the United States, $66 \%$ and $16 \%$ used Facebook and Twitter, respectively, in 2016. Eighty-three percent of Facebook users and 27\% of Twitter users are aged between 18 and 29 years; most use is on mobile devices, that comprises the vast majority of medical trainees (medical school and residency). The main age, in our study, was $27,5 \pm 2,9$ years.

Scanfeld and al, confirmed that Twitter is a space for the informal sharing of health information and advice. The dissemination of information on Twitter through networks of followers and a culture of "retweeting" demonstrate the potential reach of this medium for the dissemination of both valid and invalid information. It is therefore important for health care professionals to have a basic understanding of such services and the nature of the health-related information that is shared on them [2].

Pickering JD and al explored how a Facebook Page could support medical students in preparation for summative anatomy assessments and alleviate test anxiety. They concluded that Facebook is an emerging area of educational research [3].

With the introduction of any technology-enhanced learning resource into a curriculum, it is important to understand the role of such resources. Pickering and al provided valuable empirical evidence into the utility and benefit of integrating a social media platform into a medical anatomy curriculum.

\section{Conclusion:}

We report a positive impact of Facebook learning program in improving transfusion safety knowledge. So, SNS should be widely used as a new and efficient medical learning tool. 